\title{
Multi-scale Modeling of Deformation during Metal Forming
}

Mark R. Stoudt, Paul E. Krajewski, Michael P. Miles, and John E. Carsley

New materials are needed to meet the rapid evolution of technology in a variety of industries. Unfortunately, the Edisonian approach of testing multiple variants of a material until a suitable composition or thermo-mechanical process is identified takes too long, and results in a 7- to 10-year cycle for implementation of a fully validated, new material. Multi-scale modeling has become one of the "buzz words" to describe the ability to model properties of materials using a variety of calculations at different length scales. Much of the multi-scale research is intended to speed up the process of material design by performing virtual experiments. Multi-scale modeling is a key element of the Integrated Computational Materials Engineering initiative, ${ }^{1}$ which relies on many of these calculations to develop and validate engineering solutions.

The following set of papers provides three examples of how multi-scale modeling can be used to better understand metal deformation. The papers were selected and reviewed by the TMS Shaping and Forming Committee.

In "Atoms to Autos: A Multi-scale Approach to Modeling Aluminum Deformation," P.E. Krajewski, L.G. Hector, Jr., Y. Qi, R.K. Mishra, A.K. Sachdev, A.F. Bower, and W.A. Curtin describe the results of an extensive re- search program to develop microstructurally informed constitutive equations for aluminum sheet forming at room and elevated temperature.

In "Multi-scale Modeling of Lowtemperature Deformation in b.c.c. Metals," Thomas E. Buchheit, Corbett C. Battaile, Christopher R. Weinberger,

\section{Unfortunately, the Edisonian approach of testing multiple variants of a material until a suitable com- position or thermo- mechanical process is identified takes too long, and results in a 7- to 10-year cycle for implementation of a fully validated, new material.}

and Elizabeth A. Holm describe a multi-scale approach where atomistic information is used in single- and poly-crystalline plasticity calculations to study the effect of microstructure on yield surfaces and tensile behavior.

Finally, in "A Full-field Strategy To Take Texture-induced Anisotropy into Account During FE Simulations of Metal Forming Processes," Paul Van Houtte, Jerzy Gawad, Philippe Eyckens, Bert Van Bael, Giovanni Samaey, and Dirk Roose demonstrate a fullfield approach to incorporate complex descriptions of texture, into models, which can accurately predict earing behavior during deep drawing of sheet steel.

These papers demonstrate that while there are many approaches to "multiscale modeling," they all provide the framework to link lower length scale information with large scale calculations and solve real world engineering problems.

\section{References}

1. John Allison, Dan Backman, and Leo Christodoulou, JOM, 58 (11) (2006), pp. 25-27.

Mark R. Stoudt, research engineer, is with the National Institute of Standards and Technology (NIST), Gaithersburg, MD and is the chairman of the Shaping \& Forming Committee of the Materials Processing \& Manufacturing Division of TMS; Paul E. Krajewski, engineering group manager and technical fellow, and vice chairman and JOM advisor from the Shaping \& Forming Committee, and John E. Carsley, researcher and member of the Shaping \& Forming Committee, are with General Motors Company, Warren, MI; and Michael P. Miles, assistant professor, is with Brigham Young University, Provo, UT.

Mark R. Stoudt, Paul E. Krajewski, Michael P. Miles, \& John E. Carsley are TMS Members! To read more about them, turn to page 11. To join TMS, visit www.tms.org/Society/Membership.aspx. 